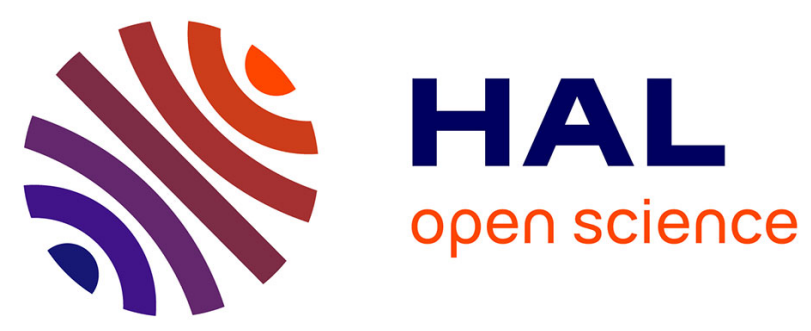

\title{
Correlation bias analysis - A novel method of sinus cardinal model for least squares estimation in cross-correlation
}

Thanh Huy Nguyen, Myriam Chabah, Christophe Sintes

\section{- To cite this version:}

Thanh Huy Nguyen, Myriam Chabah, Christophe Sintes. Correlation bias analysis - A novel method of sinus cardinal model for least squares estimation in cross-correlation. OCEANS 2015, Oct 2015, Washington, DC, United States. 10.23919/OCEANS.2015.7404526 . hal-01387453

\section{HAL Id: hal-01387453 https://hal.science/hal-01387453}

Submitted on 21 Mar 2019

HAL is a multi-disciplinary open access archive for the deposit and dissemination of scientific research documents, whether they are published or not. The documents may come from teaching and research institutions in France or abroad, or from public or private research centers.
L'archive ouverte pluridisciplinaire HAL, est destinée au dépôt et à la diffusion de documents scientifiques de niveau recherche, publiés ou non, émanant des établissements d'enseignement et de recherche français ou étrangers, des laboratoires publics ou privés. 


\title{
Correlation bias analysis - \\ A novel method of sinus cardinal model for least squares estimation in cross-correlation
}

\author{
Thanh Huy NGUYEN*, Myriam CHABAH ${ }^{\dagger}$, Christophe SINTES* \\ *TELECOM Bretagne - UMR CNRS 6285 Lab-STICC/CID - 29238 Brest Cedex 3 - France \\ †Thales Underwater Systems SAS - 29238 Brest Cedex 3 - France \\ \{thanh.nguyen, christophe.sintes\}@telecom-bretagne.eu, myriam.chabah@fr.thalesgroup.com
}

\begin{abstract}
On the subject of discrete time delay estimation (TDE), especially in the domain of sonar signal processing, in order to determine the time delays between signals received by two separate sensors, TDE techniques involve in locating the peak of cross-correlation function (CCF) between these signals. In many widely used applications of TDE, bias errors of delay estimate can occur when we try to fit the correlation function with a curve that may have an irrelevant shape, for example a parabola or a cosine. This paper thus addresses an analysis of correlation bias in estimating the time delay between a reference signal and a delayed signal by their CCF. Furthermore, we will also introduce a novel bias reduced approach for discrete TDE based on a sinus cardinal model fitting on the CCF of these two sampled signals. The experimental results have shown that the proposed method can provide relevant detection on simulated signals.
\end{abstract}

Keywords-cross-correlation function, correlation bias, discrete time delay estimation, parabolic interpolation, sinus cardinal model.

\section{INTRODUCTION}

Time delay estimation (TDE) is an important research topic in many signal processing areas, such as radar, sonar, seismology, ultrasonic, etc. It has become an interesting research issue in these fields for years with plenty of applications, especially in direction of arrival (DOA) and range estimation of array processing used in radar and sonar systems. In many cases, the time delay is estimated by comparing the signals received at two (or more) spatially separated sensors, one of them can be considered as the reference signal and the another one as the delayed signal. Classical TDE techniques consist in identifying the peak of the cross-correlation function (CCF) between these two signals.

Moreover, in case of discrete time delay estimation, it is important to take into account the effects of sampled signals but still maintain the performance of continuous signal processing on the time delay estimation. On this subject, there is an approximate analysis of the accuracy of the discrete-time cross-correlation TDE using a parabolic interpolation which has been performed in [1] [2]. However, as this conventional interpolation method may cause an undesired bias of correlation peak location estimate, furthermore in this paper, let us review the analysis of correlation bias. Besides, instead of using that quadratic interpolation approach, we also propose a sinus cardinal model which tries to fit the discrete cross correlation function by applying the non-linear least squares estimation.

The paper is organized as follows. Section II will present the TDE problem in both continuous and discrete time domains. Then, the methodology of locating the peak of crosscorrelation function will be carried out in Section III as well as the theoretical correlation bias analysis. In order to evaluate the accuracy and quality of proposed method, some experimental results will be given in Section IV. Finally, Section V will bring us the overall conclusion and perspective.

\section{THE TDE PROBLEM}

A signal emitting from a remote source and captured in the presence of noise at two spatially separated sensors can be mathematically modeled as:

$$
\begin{aligned}
& x_{1}(t)=s(t)+n_{1}(t) \\
& x_{2}(t)=A s\left(t-\tau_{0}\right)+n_{2}(t)
\end{aligned}
$$

This model of received signals refers to two differently delayed and scaled observations of the same signal $s(t)$ and two measurement noises $n_{1}(t)$ and $n_{2}(t)$ that are uncorrelated with each other and with signal $s(t)$. Let us assume that both the signal and the noises are realizations of zero-mean stationary processes characterized by their auto-correlation functions $R_{s}(\tau), R_{n_{1}}(\tau)$ and $R_{n_{2}}(\tau)$. The signal $x_{1}(t)$ will be referred to as reference signal, and the signal $x_{2}(t)$ as delayed signal. The problem is to precisely provide an estimate $\hat{\tau}_{0}$ of the true time delay $\tau_{0}$ using a finite set of samples of signals $x_{1}(t)$ and $x_{2}(t)$.

As aforementioned, in most cases, time delay estimation techniques involve the locating of the absolute extrema of the cross-correlation function or of some other statistics of the observed signals. Therefore, we assume that the delayed signal $x_{2}(t)$ is shifted and compared with a fixed portion of the reference signal $x_{1}(t)$ into the estimation window, in other words, i.e. the TDE problem is based on a constant delay assumption. The cross-correlation function between these two signals is defined as,

$$
R_{x_{1} x_{2}}(\tau)=\mathbb{E}\left[x_{1}(t) x_{2}^{*}(t-\tau)\right]
$$

where $\mathbb{E}[$.$] is the mathematical expectation and (.)* denotes$ the complex conjugate operator. An estimate of time delay will be provided by the argument $\tau$ that maximizes (2). However, in consequence of the finite observation time, only an estimate 
of the cross-correlation function $R_{x_{1} x_{2}}(\tau)$ can be obtained. Certainly, for example, assuming $x_{1}(t)$ and $x_{2}(t)$ are ergodic processes, an estimate of $R_{x_{1} x_{2}}(\tau)$ is given by,

$\hat{R}_{x_{1} x_{2}}(\tau) \doteq\left\{\begin{array}{lr}\frac{1}{T-\tau} \int_{0}^{T-\tau} x_{1}(t) x_{2}^{*}(t-\tau) d t & 0 \leq \tau<T \\ \frac{1}{T-|\tau|} \int_{|\tau|}^{T} x_{1}(t) x_{2}^{*}(t-\tau) d t & -T<\tau \leq 0\end{array}\right.$

where $T$ represents the observation interval.

On the other hand, in discrete time domain, the discrete $\mathrm{CCF}$ can be computed as,

$$
\hat{R}_{x_{1} x_{2}}(\tau) \doteq \frac{1}{N} \sum_{k=0}^{N-1} x_{1}\left(k T_{s}\right) x_{2}^{*}\left(k T_{s}-\tau\right)
$$

where $T_{s}$ is the sampling interval, $N$ is the number of samples and $(N-1) T_{s}$ is equal to the estimation window width. Nevertheless, according to this estimate of cross-correlation function, we also have a direct correlator estimator,

$$
\hat{\tau}_{0}=\underset{\tau}{\arg \max } \hat{R}_{x_{1} x_{2}}(\tau)
$$

The next section will give us an overview of the methods used in the correlation-based time delay estimation.

\section{Methodology}

Since $T_{s}$ is the sampling period and the time delay $\tau_{0}$ between two sampled signals is generally not an integer multiple of $T_{s}$, we express it into two components as,

$$
\tau_{0}=\Delta+\delta=k T_{s}+\delta
$$

The first part of (6) is an integer multiple of the sampling interval $T_{s}$. On the other hand, $\delta$ is the fractional part of the time delay $\left(0 \leq|\delta|<T_{s}\right)$. Thus, the time delay estimation can be effectuated in two steps.

- Firstly, the estimate $\hat{\Delta}$ of coarse delay is found by locating the maximum sample of (4).

- Secondly, the estimate $\hat{\delta}$ of fine delay (or subsample delay) can be computed by using a subsample interpolation technique, such as curve-fitting (parabola, cosine, spline fitting) or reconstruction-based method.

In regard to the subsample interpolation techniques which are described in [3] and [4], there are many widely used techniques that introduce significant bias errors of time delay estimate. For example, some of these techniques use the fact that the phase of analytic CCF between discrete-time signals in the vicinity of the propagation delay (group delay) will have a slope equivalent to the center frequency and will meet a zero crossing at the precise subsample shift, e.g., in [5]. Besides, the authors of [6] proposed to use a direct and computationally intensive approach to determine a precise signal shift, it consists in resampling the signals through interpolation (e.g. by a spline) before locating the cross-correlation peak. In addition, as proposed and studied in [7], performing a matched filtering (e.g. an optimum linear filter) on $\mathrm{CCF}$ may also improve the results in the case of low signal-to-noise ratio (SNR). Instead of resampling and recomputing the cross-correlation, we can also fit the $\mathrm{CCF}$ around its peak with a curve in order to reduce computational expenses. For example, a parabola (see Fig. 1) or cosine fit can be applied on a number (three or more) of samples in order to estimate the location of cross-correlation peak. The advantage of this curve-fitting method is that it is simple and easy to implement and is also computationally efficient, however, it suffers from bias errors because the underlying signal may mismatch the chosen curve.

Fortunately, according to [1] and [3], the curve-fitting bias errors can be avoided by applying a sinus cardinal interpolating function on discrete cross-correlation, which is the maximum likelihood estimation of the time delay. The authors of [3] examined the use of sinus cardinal reconstruction to locate the cross-correlation peak and found that it significantly outperforms parabolic or cosinusoidal interpolation. However, this reconstruction is highly computationally expensive in comparison with curve-fitting methods. Therefore, a numerical optimization needs to be implemented to decrease computation times in the peak locating, e.g. a binary search method.

\section{A. Conventional curve-fitting interpolation method}

As aforementioned, in discrete TDE, time delay between two sampled received signals usually is not an integer multiple of the sampling period. Thus, in order to perform an analog processing of the time delay parameter, an approximation which is simple and widely used consists in fitting a curve, for example a convex upward parabola (or other polynomials) or a cosine in the vicinity of the correlation peak.

$$
\hat{R}_{x_{1} x_{2}}(\tau)=a \tau^{2}+b \tau+c
$$

where $a, b$ and $c$ (with $a<0$ ) are the parabolic parameters fitting the measured cross-correlation function, and the values $\tau$ are in the neighborhood of the correlation peak. This parabolic fit can be guaranteed perfectly due to three samples, which are the peak of the discrete correlation function and its two neighboring samples.

Furthermore, an explicit expression exists for locating the peak value as the vertex of parabola,

$$
\hat{\delta}=-\frac{b}{2 a}
$$

However, this parabolic interpolation approach, as other curvefitting interpolations (e.g. cosine fitting, spline fitting) always yield biased estimates of the time delay [1][2][3][6], affecting the use of these methods in some applications.

Indeed, given the largest sample $y_{1}$ of cross-correlation function $\hat{R}_{x_{1} x_{2}}$, which occurs at instant $\Delta$, and its two nearest neighbors $y_{0}, y_{2}$ at instants $\Delta-T_{s}$ and $\Delta+T_{s}$. As a parabola is a quadratic function that only needs three parameters as $a, b$ and $c$ to be identically determined, it is hence possible to determine the parabola peak location via its three largest samples $\left(y_{0}, y_{1}, y_{2}\right)$. Continuing the second step of the discrete time delay estimation, as aforestated from (6), the estimate $\hat{\delta}$ of sub-sample shift can be found by fitting a parabola to these three samples of $\hat{R}_{x_{1} x_{2}}(\tau)$ function about $\Delta$.

$$
\hat{\delta}=-\frac{b}{2 a}=-\frac{y_{2}-y_{0}}{2\left(y_{2}-2 y_{1}+y_{0}\right)}
$$




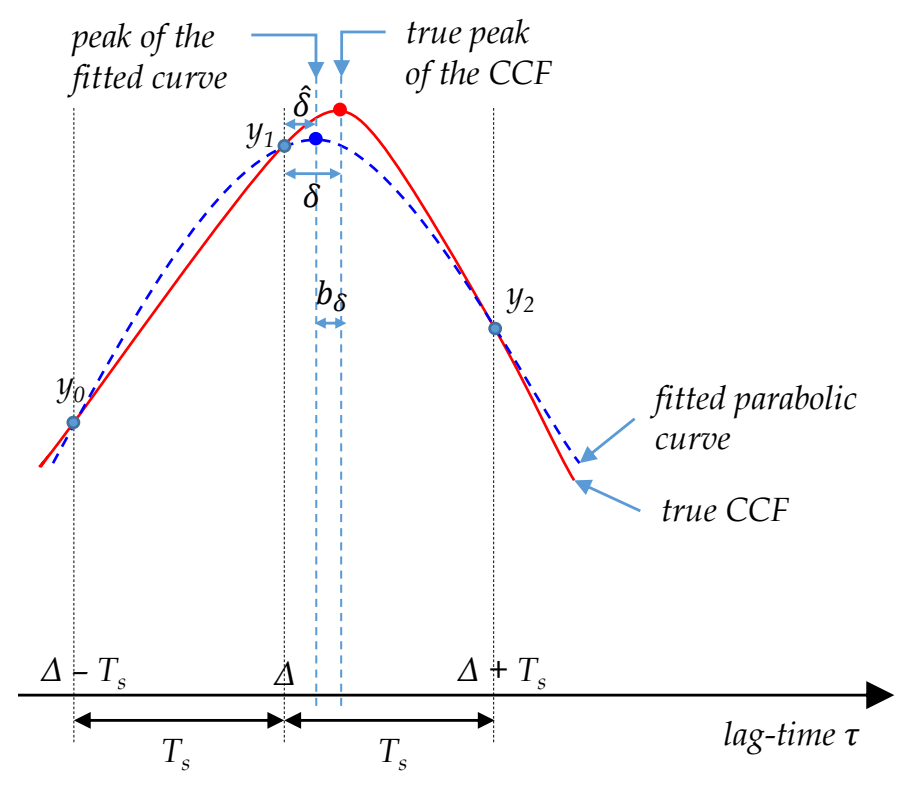

Fig. 1. An illustration of the parabolic fitting on three largest samples $\left(y_{0}, y_{1}, y_{2}\right)$ of $\mathrm{CCF}$, as well as the bias error $b_{\delta}$ of estimate of the correlation peak location in comparing the peak from fitted parabola (blue dashed curve) and the peak of the true CCF (red solid curve)

The estimate $\hat{\delta}$ can be expressed in terms of finite differences. The numerator is the average of two first differences,

$$
d(\delta)=\frac{1}{2}\left[d_{1}(\delta)+d_{-1}(\delta)\right]
$$

with $d_{1}(\delta)=y_{2}-y_{1}$ and $d_{-1}(\delta)=y_{1}-y_{0}$

and the denominator is equal to the second difference,

$$
d_{2}(\delta)=-\left[d_{1}(\delta)-d_{-1}(\delta)\right]
$$

Therefore, (8) can be expressed as,

$$
\hat{\delta}=\frac{d(\delta)}{d_{2}(\delta)}
$$

In addition, according to [1], the statistics of $d(\delta)$ and $d_{2}(\delta)$ can be measured via the frequency domain based on WienerKhintchine theorem. Moreover, under several assumptions declared in [1], the mean of the estimate $\hat{\delta}$ is obtainable from,

$\mathbb{E}[\hat{\delta}] \simeq \frac{\mathbb{E}[d]}{\mathbb{E}\left[d_{2}\right]}=\frac{\frac{1}{N} \sum_{k=-N / 2+1}^{N / 2-1} A G_{s}(k) \sin \left(\frac{2 \pi k \delta}{N}\right) \sin \left(\frac{2 \pi k}{N}\right)}{\frac{1}{N} \sum_{k=-N / 2+1}^{N / 2-1} A G_{s}(k) \cos \left(\frac{2 \pi k \delta}{N}\right)\left[2-2 \cos \left(\frac{2 \pi k}{N}\right)\right]}$

where $G_{s}(k)$ is the power spectral density of the zero-mean stationary Gaussian process $s(k)$, and $A$ is the scale factor of the replica $x_{2}$ versus signal $s$ which appeared in (1).

Hence, the bias error of estimate is defined as,

$$
b_{\delta}=\mathbb{E}[\hat{\delta}]-\delta
$$

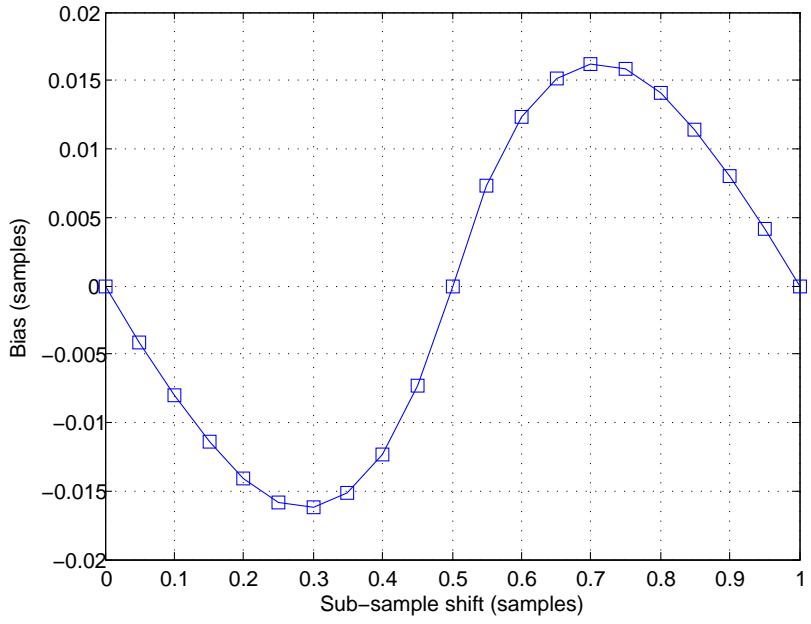

(a) Theoretical bias error

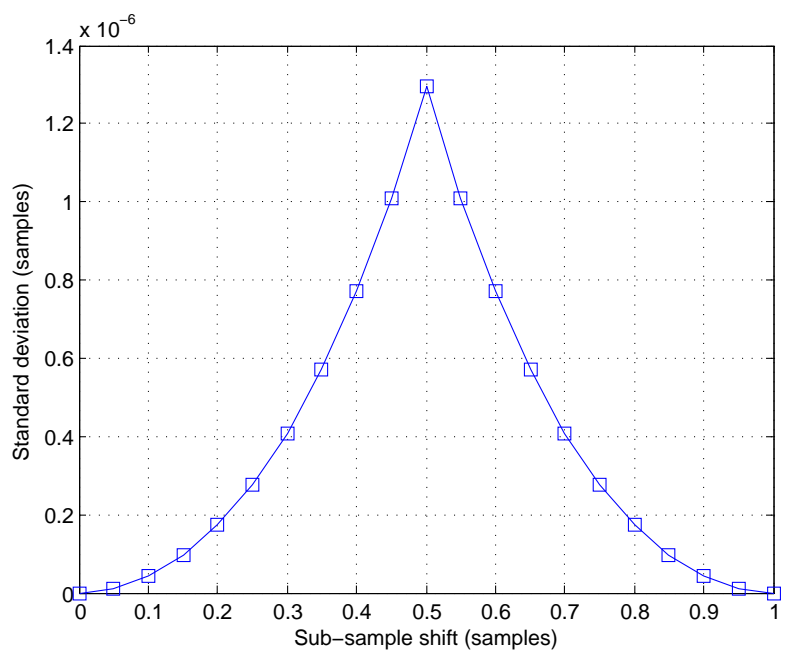

(b) Theoretical standard deviation

Fig. 2. Theoretical bias error and standard deviation of delay estimate (parabolic interpolation method)

Besides, the standard deviation of estimate $\hat{\delta}$ is given by,

$$
\begin{aligned}
& \sigma_{\hat{\delta}}^{2} \simeq \frac{\sigma_{d(\delta)}^{2}}{\mathbb{E}^{2}\left[d_{2}(\delta)\right]} \\
& =\frac{\frac{1}{N^{2}} \sum_{k=-N / 2+1}^{N / 2-1} G_{x_{1}}(k) G_{x_{2}}(k) \sin ^{2}\left(\frac{2 \pi k}{N}\right)\left[1-C_{12}(k) \cos \left(\frac{4 \pi k \delta}{N}\right)\right]}{\left[\frac{1}{N} \sum_{k=-N / 2+1}^{N / 2-1} A G_{s}(k) \cos \left(\frac{2 \pi k \delta}{N}\right)\left[2-2 \cos \left(\frac{2 \pi k}{N}\right)\right]\right]_{(12)}^{2}}
\end{aligned}
$$

where $C_{12}=\frac{\left|G_{x_{1} x_{2}}(k)\right|^{2}}{G_{x_{1}}(k) G_{x_{2}}(k)}$, and $G_{x_{1}}(k)$ (resp., $\left.G_{x_{2}}(k)\right)$ is the power spectral density of signal $x_{1}$ (resp., signal $x_{2}$ ).

Equations (10) and (12) predict the performance of parabolic interpolation to locate the correlation peak in time delay estimation. Moreover, Fig. 2a and 2b illustrate the theoretical bias and standard deviation of time delay estimate in a noiseless case. 


\section{B. Proposed method}

Since the curve-fitting methods might cause undesired bias of delay estimate, it is necessary to perform an unbiased delay estimate method. Studies in [3] and [4] state that a method of reconstructive interpolation can yield an unbiased estimation of discrete time delay by using a sinus cardinal function based on analog signal reconstruction theory. The interpolated samples are obtained when we perform an ideal low-pass filter by windowing a sinc-shaped impulse response with a finite window function (e.g. a Blackman window).

$$
x(t)=\sum_{k=-N / 2}^{N / 2} x_{d}(k) \operatorname{sinc}\left[\frac{\pi\left(t-k T_{s}\right)}{T_{s}}\right] w_{h}\left[\frac{\pi\left(t-k T_{s}\right)}{T_{s}}\right]
$$

where $x_{d}(k)$ is the discrete signal, $\operatorname{sinc}(t)=\sin (t) / t$ and $w_{h}$ is the Blackman window.

This reconstructive interpolation method was studied and it is demonstrated in [3] that it yields an unbiased estimator of time delay.

On the other hand, assuming that the auto-correlation function of a signal $s(t)$ is expressed as the autocorrelation of a band-limited white noise,

$$
R_{s}(\tau)=R_{s}(0) \frac{\sin (2 \pi B \tau)}{2 \pi B \tau}
$$

where $B$ is the noise bandwidth.

Based on this property of band-limited white noise $[8, \mathrm{Sec}$. 8.4], we propose to use a sinus cardinal model to minimize errors between real data from cross-correlation function and the fitting model. A sinus cardinal function is defined with its parameters as,

$$
f(t)=A_{0} \frac{\sin \left(\alpha\left(t-\tau_{0}\right)\right)}{\alpha\left(t-\tau_{0}\right)}
$$

with $A_{0}>0$ is the maximum amplitude of the cross-correlation, and $\tau_{0}$ is its centroid, and $\alpha$ is the coefficient corresponding to the width of its principal lobe. Given a data set of $M$ points of the CCF in the vicinity of its principal and secondary lobes, $\left\{\left(t_{i}, y\left(t_{i}\right)\right), i=0, . ., M-1\right\}$, where $y\left(t_{i}\right)$ is the observation of cross-correlation function at instant $t_{i}$, this estimator aims at solving the least squares problem,

$$
\left(\hat{A}_{0}, \hat{\alpha}, \hat{\tau}_{0}\right)=\underset{A_{0}, \alpha, \tau_{0}}{\arg \min } \sum_{i=0}^{M-1}\left[y\left(t_{i}\right)-A_{0} \frac{\sin \left(\alpha\left(t_{i}-\tau_{0}\right)\right)}{\alpha\left(t_{i}-\tau_{0}\right)}\right]^{2}
$$

The objective of this novel method is to symmetrize the crosscorrelation function in order to reduce or remove the bias of estimate. Given optimal set of parameters $\left(\hat{A}_{0}, \hat{\alpha}, \hat{\tau}_{0}\right)$, the cross-correlation function is then estimated as,

$$
\hat{y}\left(t_{i}\right)=\hat{A}_{0} \frac{\sin \left(\hat{\alpha}\left(t-\hat{\tau}_{0}\right)\right)}{\hat{\alpha}\left(t-\hat{\tau}_{0}\right)}
$$

For implementation, one could consider employing LevenbergMarquardt algorithm [9] to solve the non-linear least squares problem (16). Moreover, the estimate $\hat{\tau}_{0}$ of the centroid of sinus cardinal function, in other words, the estimated location of correlation peak is the time delay between the reference

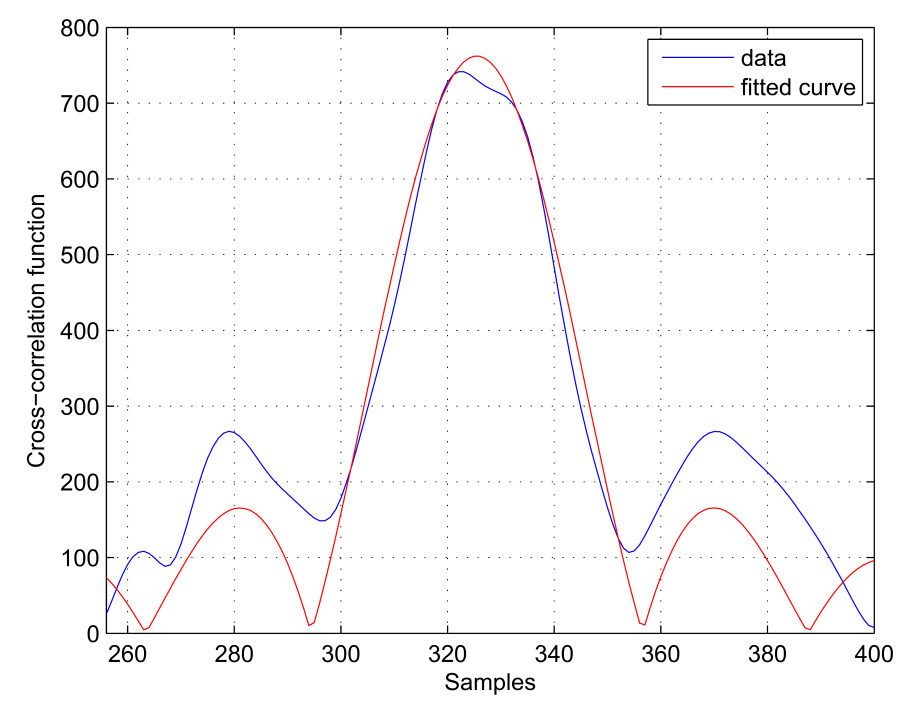

Fig. 3. Fitness of the model function - An example of the cross-correlation function around its principal and secondary lobes with the fitted sinus cardinal using the model function in (15).

and delayed signals. Fig. 3 depicts an example of the crosscorrelation function at its principal and secondary lobes and the fitted sinus cardinal curve from the aforementioned nonlinear least squares method. The correlation function has been shown to be well-fitted by the model function (15), especially in the considered region around the principal lobe.

\section{EXPERIMENTAL RESULTS}

\section{A. Sonar signal simulator}

TABle I. Sonar Signal Simulator Parameters

\begin{tabular}{|c|c|c|}
\hline Bandwidth & Carrier frequency & Sampling period \\
$B$ & $f_{0}$ & $T_{s}$ \\
\hline $20 \mathrm{kHz}$ & $100 \mathrm{kHz}$ & $12.5 \mu s$ \\
\hline
\end{tabular}

The sonar signals are computed by the complex summation of elementary backscatterers contained within the resolution cell. Thus, the backscattered signal received by each sensor can be modeled by:

$$
\begin{aligned}
& s_{1}\left(k T_{s}\right)=\sum_{m \in\left[a_{k}\right]} R_{m} e^{j \phi_{m}} e^{j 2 \pi f_{0}\left(k T_{s}-m \lambda\right)} \mathbf{1}_{\left[0 T_{s}\right]}\left(k T_{s}-m \lambda\right) \\
& s_{2}\left(k T_{s}\right)=\sum_{n \in\left[b_{k}\right]} R_{n} e^{j \phi_{n}} e^{j 2 \pi f_{0}\left(k T_{s}-n \lambda-\tau_{0}\right)} \mathbf{1}_{\left[0 T_{s}\right]}\left(k T_{s}-n \lambda-\tau_{0}\right)
\end{aligned}
$$

where

- $\left[a_{k}\right]$ and $\left[b_{k}\right]$ stand for the resolutions cell of each sensor $a$ and $b$ at an instant $k$, which contain many elementary backscatterers inside;

- $\lambda$ denotes the time shift between one brillant point to the next one;

- $\quad \mathbf{1}_{X}(x)$ is the indicator function, which results 1 if $x \in$ $X$ and results 0 if $x \notin X$.

Moreover, the reflectivity of elementary backscatterers is distributed as a circular Gaussian process.

$$
R_{n} e^{j \phi_{n}} \sim \mathcal{C N}\left(0, \sigma^{2}\right)
$$




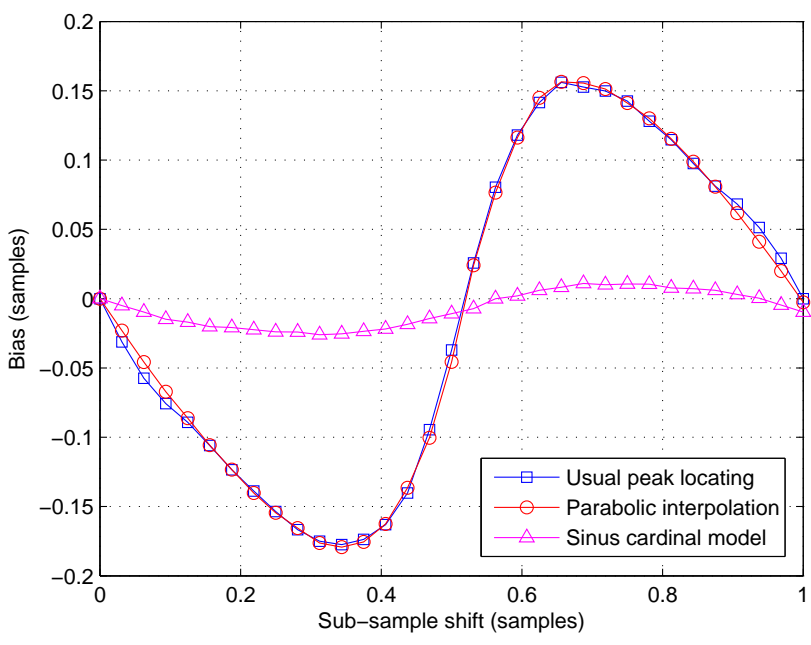

(a) Bias error of delay estimation

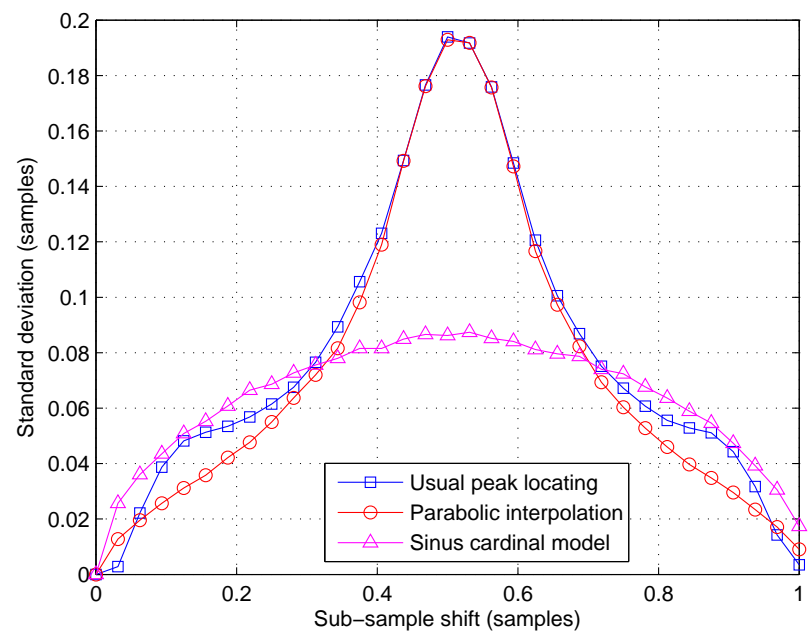

(b) Standard deviation of delay estimation

Fig. 4. Bias error and standard deviation of delay estimation among methods in the noiseless case (5000 independent Monte-Carlo realizations)

where $R_{n}$ has a Rayleigh distribution of parameter $\sigma^{2}$, and $\phi_{n}$ is a uniform random variable distributed on a $2 \pi$-length interval.

\section{B. Application of TDE methods on sonar simulated signals}

Based on the sampled signals simulated from a sonar signal simulator, we are able to experiment and evaluate the bias and standard deviation of the proposed method as well as the conventional method. Besides, the bias and standard deviation curves of the ordinary cross-correlation peak locating using oversampling (by a factor of 10) on CCF are also illustrated alongside with two mentioned methods in order that one may compare them.

Fig. $4 \mathrm{a}$ and $4 \mathrm{~b}$ depict the bias and the standard deviation of discrete time delay estimation for different methods as a function of the sub-sample shift between the reference and delayed signals. For each shift value, 5000 independent Monte Carlo tests were realized. As the bias and standard deviation

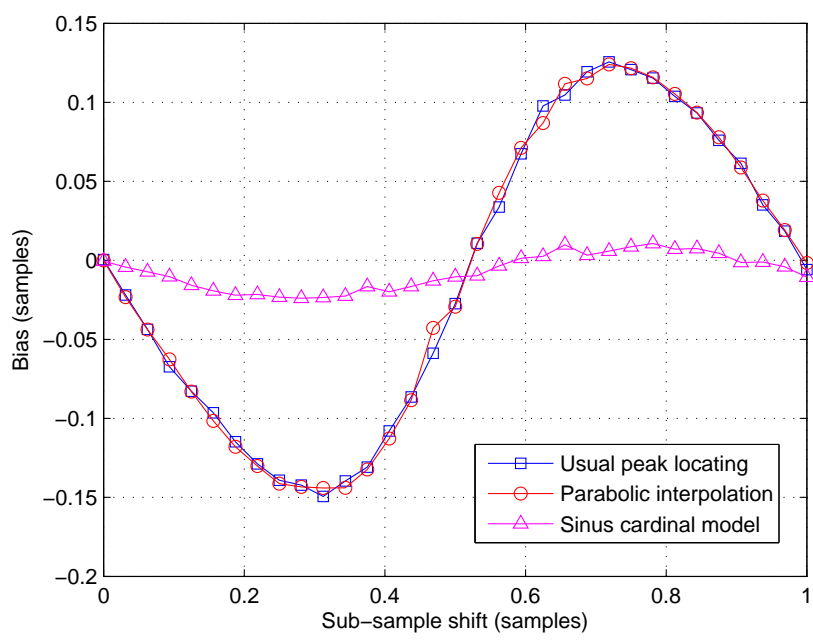

(a) Bias error of delay estimation

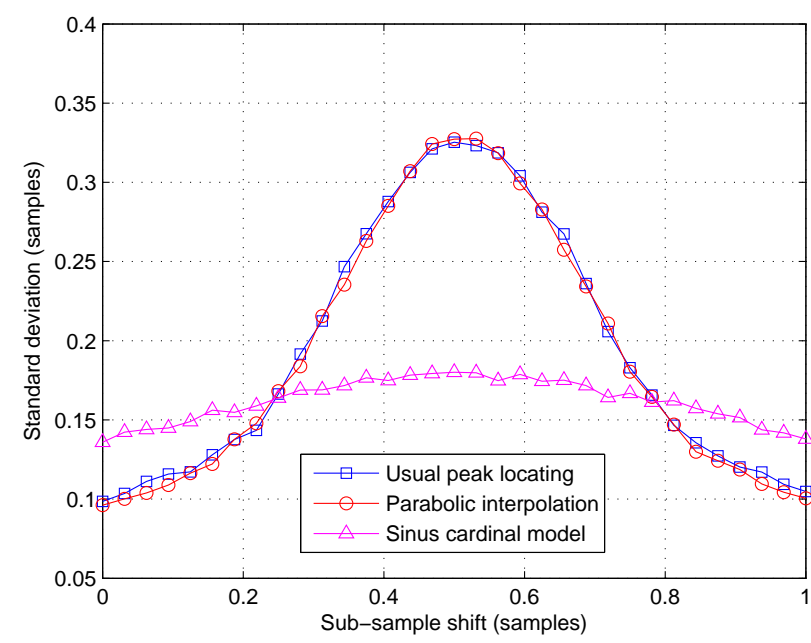

(b) Standard deviation of delay estimation

Fig. 5. Bias error and standard deviation of delay estimation among methods in the noisy case SNR $=-15 \mathrm{~dB}$ (5000 independent Monte-Carlo realizations)

of the proposed model have been significantly reduced in comparison with other methods, the results for simulated sonar signals satisfy our first goal and make this method become very promising in many TDE applications. Moreover, in the presence of additive Gaussian white noise (SNR $=-15 \mathrm{~dB}$ ), the Monte Carlo realizations have also been launched in order to evaluate the quality of these methods in a noisy case (see Fig. 5).

\section{CONCLUSION AND PERSPECTIVES}

This paper has demonstrated again that the bias error of the curve-fitting interpolation methods is a function of the spectral characteristics of the signals received. Different methods of time delay estimation have been analysed and tested on simulated sonar signals, including curve-fitting interpolation and sinus cardinal kernel method. The bias and standard deviation of time delay estimate have been given as a function of subsample shift, showing that the proposed method using sinus 
cardinal model achieves a significant bias reduction.

On the one hand, the main advantage of curve-fitting interpolation is its computational simplicity, this makes it the method of choice in TDE applications where the bias error that results from interpolations is suboptimal and acceptable. On the other hand, bias error reduction is an advantage of sinus cardinal kernel method. This proposed method will be relevant and useful in many further TDE applications in the future.

\section{REFERENCES}

[1] R. E. Boucher and J. C. Hassab, "Analysis of discrete implementation of generalized cross correlator," Acoustics, Speech and Signal Processing, IEEE Transactions on, vol. 29, no. 3, pp. 609-611, 1981.

[2] G. Jacovitti and G. Scarano, "Discrete time techniques for time delay estimation," Signal Processing, IEEE Transactions on, vol. 41, no. 2, pp. 525-533, 1993.

[3] I. Céspedes, Y. Huang, J. Ophir, and S. Spratt, "Methods for estimation of subsample time delays of digitized echo signals," Ultrasonic imaging, vol. 17, no. 2, pp. 142-171, 1995.

[4] M. M. McCormick and T. Varghese, "An approach to unbiased subsample interpolation for motion tracking," Ultrasonic imaging, vol. 35, no. 2, pp. 76-89, 2013.

[5] J. Marple, S.L., "Estimating group delay and phase delay via discretetime analytic cross-correlation," Signal Processing, IEEE Transactions on, vol. 47, no. 9, pp. 2604-2607, Sep 1999.

[6] F. Viola and W. F. Walker, "A spline-based algorithm for continuous time-delay estimation using sampled data," Ultrasonics, Ferroelectrics, and Frequency Control, IEEE Transactions on, vol. 52, no. 1, pp. 80-93, 2005.

[7] X. Lai and H. Torp, "Interpolation methods for time-delay estimation using cross-correlation method for blood velocity measurement," Ultrasonics, Ferroelectrics, and Frequency Control, IEEE Transactions on, vol. 46, no. 2, pp. 277-290, March 1999.

[8] J. S. Bendat and A. G. Piersol, Random data: analysis and measurement procedures. John Wiley \& Sons, 2011, vol. 729.

[9] D. W. Marquardt, "An algorithm for least-squares estimation of nonlinear parameters," Journal of the Society for Industrial \& Applied Mathematics, vol. 11 , no. 2, pp. 431-441, 1963. 